\title{
Les associations chrétiennes de Damas et leurs rapports variables au religieux (2000-2010)
}

Christian Associations in Damascus and their Fluctuant Relations to Religion (2000-2010)

Las asociaciones cristianas de Damasco y sus relaciones variables con lo religioso (2000-2010)

Laura Ruiz de Elvira

\section{(2) OpenEdition}

\section{Journals}

Édition électronique

URL : http://journals.openedition.org/assr/27071

DOI : $10.4000 /$ assr. 27071

ISSN : $1777-5825$

Éditeur

Éditions de l'EHESS

Édition imprimée

Date de publication : 1 septembre 2015

Pagination : 121-138

ISBN : 9-782713224706

ISSN : 0335-5985

\section{Référence électronique}

Laura Ruiz de Elvira, «Les associations chrétiennes de Damas et leurs rapports variables au religieux (2000-2010) », Archives de sciences sociales des religions [En ligne], 171 | 2015, mis en ligne le 01 septembre 2018, consulté le 22 avril 2019. URL : http://journals.openedition.org/assr/27071 ; DOI 10.4000/assr. 27071 


\section{Laura Ruiz de Elvira}

\section{Les associations chrétiennes de Damas et leurs rapports variables au religieux (2000-2010)}

Bien que les travaux consacrés à l'histoire et à la situation contemporaine des chrétiens du Proche-Orient soient abondants (J.-P. Valognes, 1994 ; G. Chatelard, 2004 ; S. Griffith, 2008 ; F. Pichon, 2010 ; F. McCallum, 2010), la vie quotidienne des membres de ces communautés ${ }^{1}$, leurs loisirs et les formes de leur sociabilité, ainsi que leurs modalités d'organisation sociale et d'action collective sont peu connus. De même, peu de travaux se sont attachés à analyser en détail les rapports variables au religieux des chrétiens arabes de notre temps qui, dans le cas de la Syrie prérévolutionnaire, représentaient autour de $10 \%$ de la population. Pourtant, à nos yeux, ces rapports demeurent centraux si l'on veut comprendre les dynamiques et l'identité même de ces communautés.

Ainsi, l'objectif de cet article est d'explorer ces rapports au travers de l'étude de plusieurs associations chrétiennes à vocation sociale qui travaillaient dans la capitale syrienne au cours des années 2000. Il faut préciser ici que nous considérerons comme "chrétienne " toute association qui remplit au moins l'une des conditions suivantes : être sous le patronage direct ou indirect d'une institution religieuse chrétienne ; avoir son siège dans un bâtiment appartenant à une institution religieuse chrétienne ; avoir été fondée par un membre du clergé chrétien ; porter un nom qui se réfère au christianisme ; avoir un conseil d'administration composé d'au moins un clerc chrétien ; et enfin, plus généralement, toute association qui se présente elle-même comme chrétienne. Il faut néanmoins garder à l'esprit que l'identité religieuse d'une organisation est avant tout subjective, « elle repose sur l'auto-identification comme telle par l'ONG elle-même et non sur un corpus de normes extérieures qui la définiraient ainsi de manière objective " (J. Berger, $2007: 28$ ).

Il s'agira de montrer in fine que, malgré le recours fréquent à une rhétorique insistant sur leur caractère aconfessionnel (c'est-à-dire non religieux) et noncommunautaire, ces structures associatives entretiennent un rapport étroit et

1. Les chrétiens de Syrie sont divisés en onze communautés différentes. 
profond à la religion chrétienne et s'appuient sur des sociabilités et réseaux communautaires chrétiens aussi bien locaux et nationaux qu'internationaux. Pour ce faire, l'analyse sera développée en cinq points. Nous reviendrons tout d'abord sur le moment de leur fondation, qui est souvent en lien avec des institutions religieuses chrétiennes. Nous poursuivrons par l'étude de leur statut légal, ce qui nous permettra de mettre au jour la manière dont les liens noués avec les différentes Églises lors de la fondation se sont prolongés dans le temps. Nous nous pencherons ensuite sur leur supposé caractère aconfessionnel et non-communautaire ainsi que sur la façon dont celui-ci est mis à l'épreuve par les sociabilités, les pratiques sociales et l'ethos (P. Bourdieu, 1984/2002) des acteurs qui y sont engagés. Puis, nous illustrerons, à travers quelques exemples, comment les références à la religion sont de fait récurrentes au sein de ces associations. Enfin, nous mettrons en lumière leur insertion, par le biais de réseaux plus ou moins formels, dans les tissus associatifs chrétiens internationaux, très actifs et bénéficiant d'une forte visibilité.

Cette étude aborde, bien que de manière indirecte, la question de la gestion de la diversité confessionnelle par le pouvoir syrien sous le régime de Hafez alAssad et celui de son fils Bachar depuis 2000 et montre, en filigrane, la relation privilégiée des acteurs sociaux et religieux chrétiens avec les détenteurs du pouvoir politique dans la Syrie baathiste (L. Ruiz de Elvira, sous presse). Il faut rappeler ici que si le régime syrien s'est toujours présenté comme laïc et comme garant d'une cohabitation pacifique entre des communautés ethniques et confessionnelles diverses, il a également su jouer des divisions communautaires et instrumentaliser les minorités pour rester au pouvoir (Z. Taha, 2012).

Les associations à vocation sociale constituent la pierre de touche d'une société civile syrienne relativement méconnue avant 2011, que ce soit dans sa composante chrétienne, musulmane ou séculière, malgré son épanouissement et son renouvellement au cours des années 2000 (L. Ruiz de Elvira, 2010). Seuls quelques articles font figure d'exception (S. Boukhaima, 2002; M. Le Saux, 2006 ; T. Pierret et K. Selvik, 2009 ; L. Ruiz de Elvira et T. Zintl, 2012). Ces recherches s'inscrivent dans la lignée d'autres travaux scientifiques qui se sont multipliés à partir des années 1990, et qui ont cherché à mettre en lumière les modalités de l'essor des sociétés civiles arabes et musulmanes, d'une part, et leur place, leur nature, leurs rôles et fonctions, d'autre part (R. Norton, 1995 ; S. Ben Nefissa, 2002 ; S. Hashmi, 2002 ; A. Bozzo et P.-J. Luizard, 2011). Les mouvements révolutionnaires $\mathrm{du}$ " printemps arabe » n’ont fait que souligner la nécessité et l'importance de ce type de travaux.

Partant d'une démarche de sociologie politique des acteurs, des discours et des pratiques du secteur associatif syrien, notamment caritatif, nous nous appuierons sur une investigation de deux années (menée d'octobre 2007 à janvier 2010) au cours de laquelle vingt-neuf associations syriennes, dont dix chrétiennes, ont été visitées régulièrement et près d'une soixantaine d'entretiens semi-directifs ont 
été réalisés avec différents acteurs impliqués dans le monde associatif et le secteur de la bienfaisance. Au-delà des entretiens, notre travail se fonde également sur l'observation participante réalisée dans différentes associations chrétiennes, dont Terre des Hommes Syrie, Bayt al-Salām et al-Safīna, où nous avons pris part à plusieurs activités (par exemple au sein des ateliers de production, du camp de vacances de Kfarseta et des marchés caritatifs de Noël). Ainsi, nous avons pu observer aussi bien des moments de vie quotidienne que des situations plus exceptionnelles. Ce travail de terrain a été complété par la lecture de documents produits par ces organisations (brochures, rapports internes, etc.).

L'ensemble des logiques et des situations analysées ici est antérieur au déclenchement du processus révolutionnaire de 2011, processus dont on ne peut prévoir ni l'issue ni l'impact (tant sur l'ensemble du pays que sur les populations chrétiennes) au moment où nous écrivons ces lignes. Les rares informations que l'on peut obtenir à travers Internet, complétées par des entretiens menés en Turquie et au Liban au printemps 2014, semblent néanmoins indiquer que les associations chrétiennes étudiées dans cet article survivent mieux au conflit que leurs homologues sunnites. En effet, alors que la plupart des structures sunnites que nous avions visitées entre 2007 et 2010 ont soit fermé leurs locaux soit diminué fortement leurs activités, les associations chrétiennes de Damas continuent, elles, d'élire leur conseil d'administration, de collecter des fonds, de célébrer des fêtes et de mener leurs activités quotidiennes. En ces temps de violence extrême, elles contribuent, et peut-être plus que jamais, à préserver et régénérer les liens sociaux.

\section{Des fondations en lien avec des institutions religieuses}

La première association formelle créée sur le territoire qui correspond à la Syrie actuelle est l'association chrétienne, et plus précisément grecque-melkite catholique, Saint Vincent de Paul $(1863)^{2}$, qui était encore très active dans les années 2000. Sa naissance à Damas est directement liée au mouvement religieux international homonyme, lancé en France en 1833 par Frédéric Ozanam (G. Cholvy, 2003), et aux massacres de juillet 1860, qui firent plusieurs milliers de victimes et d'émigrés parmi les chrétiens de Damas. Ceci explique la nature de ses premières activités, qui consistaient notamment à visiter et secourir les survivants de ces massacres ainsi qu'à prendre en charge les veuves, les orphelins et les pauvres victimes de ces évènements. Fondée sous les auspices du patriarche de l'Église grecque-melkite, Gregorius Yussef, par un groupe de riches commerçants chrétiens habitant la capitale syrienne, et présidée pendant sa première décennie par Antūn Sakakīnī (Association de Bienfaisance Saint Vincent de Paul

2. Une association Saint Vincent de Paul a été créée au Liban en 1860. Outre le Liban et la Syrie, la Société de Saint Vincent de Paul s'est aussi installée en Algérie, en Jordanie, en Turquie et en Irak. 
de Damas, 2003), cette première branche locale de Saint Vincent de Paul sera suivie, postérieurement, par de nouvelles antennes créées dans d'autres villes du pays : Alep, Hassaké (1931), Ra’s al-'Ayn et Homs.

Près d'un siècle plus tard (1967), Terre des Hommes Syrie voit aussi le jour à Damas, puis ouvre deux antennes à Alep et Lattaquié $(1978)^{3}$. Cette association se déclare au service des enfants en situation de handicap physique et mental, des réfugiés vulnérables et des familles syriennes nécessiteuses. Le père Paul Sleiman, lazariste d'origine libanaise, installé en Syrie depuis lors, décrit la genèse de cette structure que lui-même a fondée : «1966: I was assigned as a director of the secondary department in the Vincentian school in Damascus ${ }^{4}$, and the idea of constituting a national group for charity work was born. I started working on my message, encouraged from my ministry (the Vincentian order), especially the monk Youssef Maaloly, who helped me to insight the Syrian society [...] » (Terre des Hommes Syrie, document non daté). Mais ce n'est qu'avec l'éclatement de la Guerre des Six jours, en 1967, que l'association devient vraiment active : " The war of 5 August [sic] took place, and we worked for helping 1.160 refugees families from al-Golan who lived in tents in Barzee [...], helped with the Syrian scouting mission, the Marian brotherhood, and local street soccer team, and thus the Lazarist monastery became an active hive welcoming and helping the refugees, and we used the monastery dishes and battles and other tools because "God want $[s i c]$ mercy not sacrifice" and he will be glorified by humans respect, not by stones " (Terre des Hommes Syrie, document non daté). Ces extraits de récits rétrospectifs mettent clairement en évidence le lien entre la naissance de Terre des Hommes Syrie et l'Église catholique via le père Sleiman et son ordre religieux. Ainsi Terre des Hommes Syrie, du moins à ses débuts, est une association motivée par la foi.

La création d'al-Mahabba (la Charité, 1980) est aussi étroitement liée à une institution religieuse chrétienne. Selon les propos d'une de ses fondatrices ${ }^{5}$, elle est née sous les auspices de l'Église grecque-melkite, comme dans le cas de Saint Vincent de Paul, et plus précisément avec l'aide de Monseigneur Joseph. C'est en effet autour des messes célébrées par ce clerc qu'un groupe de femmes s'est constitué. L'une d'entre elles ayant fait la connaissance d'une association travaillant pour les personnes handicapées au cours d'un voyage au Liban, eut l'idée de fonder al-Maḥabba à Damas. Grâce au soutien de Monseigneur Joseph, qui dirige dans cette période l'organisation catholique Caritas ${ }^{6}$ en Syrie, mais aussi

3. Pour une analyse exhaustive de cette association voir L. Ruiz de Elvira, 2012b.

4. Sur la fondation et l'histoire du collège Saint-Vincent des pères lazaristes de Damas voir J. Bocquet, 2002.

5. Entretien en langue française, Damas, avril 2008.

6. La première association Caritas a été fondée à Fribourg-en-Brisgau, en Allemagne, en 1897. En 1967 Caritas s'installe à Jérusalem, en Jordanie et en Syrie. Aujourd'hui cette organisation à but caritatif est présente dans 198 pays et territoires. 
des archevêques François Abū Mūh (archevêque de Palmyre) et Isidor Bațțīha (archevêque de Homs) et de l'appui de la communauté grecque-melkite, l'association peut voir le jour et se développer progressivement. En 2010, al-Mahabba était installée dans une grande maison traditionnelle située dans la vieille ville de Damas. Son activité se concentrait autour de deux programmes. Le premier consistait à accueillir pendant la semaine une quarantaine d'enfants handicapés de 5 à 25 ans. Les moniteurs œuvraient à développer leurs capacités psychomotrices et mentales par le biais d'activités variées. Le deuxième, qui prenait également en charge une quarantaine de bénéficiaires, avait pour but de leur apprendre un métier ou une activité manuelle (la couture, par exemple).

Bayt al-Salām (1992) ${ }^{7}$, quant à elle, est une petite structure associative située à quelques cent mètres d'al-Mahabba, en plein cœur de la vieille ville de Damas. Fondée sous le patronage du patriarcat syriaque, elle est née de l'initiative d'un prêtre issu de cette même communauté religieuse, qui réside désormais en Italie. Elle a pour vocation la prise en charge de personnes adultes atteintes d'un handicap mental. D'après celui qui était président du conseil d'administration en 2010, au moment où Bayt al-Salām a été fondée, aucune assistance sociale n'était accordée par les services publics et très peu d'organisations s'intéressaient aux personnes handicapées. Celles-ci restaient enfermées chez elles par leurs propres familles qui avaient souvent honte d'elles. Dans ce contexte, Bayt al-Salām avait pour objectif fondamental « que ces personnes apprennent quelque chose, qu'elles se sentent valorisées et utiles et qu'elles ne restent pas confinées dans leurs foyers " ${ }^{8}$. Quant à ceux dont le handicap était moins important, il s'agissait de leur apprendre un métier en vue de leur trouver par la suite un travail rémunéré considéré comme normal.

Ce même projet se trouve à l'origine d'al-Safīna (littéralement "l'Arche »), une association chrétienne très proche - de par ses valeurs, ses pratiques et son emplacement géographique - de Bayt al-Salām. Inspirée du projet international "l'Arche ", dont la première communauté est créée en France en 1964 par le Canadien Jean Vanier, une Syrienne, qui avait déjà travaillé dans cette communauté dans un autre pays, conçut l'idée de fonder al-Safīna en Syrie. Après un premier échec à Alep, al- Safīna ouvre finalement ses portes à Damas en 1995, sous le patronage du vicariat latin de Syrie ${ }^{9}$ et avec l'aide du prêtre jésuite Frans van der Lugt ${ }^{10}$. En 2010, l'association était installée dans deux maisons traditionnelles de la vieille ville, propriétés de l'Église latine. Son activité quotidienne s'organisait autour de deux projets : «le Foyer », qui hébergeait sept personnes handicapées qui avaient été abandonnées ou rejetées par leurs familles, et «le Port », un atelier de jour accueillant une vingtaine de personnes handicapées.

\footnotetext{
7. Pour une analyse détaillée de cette association voir L. Ruiz de Elvira, 2012a.

8. Entretien avec la directrice de l'association, Damas, décembre 2009.

9. Pour plus d'informations voir sa page web : http://www.larchesyria.com/fr/the-community/.

10. Ce jésuite hollandais a été assassiné le 7 avril 2014 à Homs.
} 
Ces cinq exemples nous permettent d'illustrer une logique plus large de création d'associations en lien avec des institutions religieuses chrétiennes. Cette logique concerne non seulement la majorité des structures chrétiennes que nous avons pu visiter à Damas, mais aussi des associations chrétiennes syriennes que l'on peut trouver dans d'autres provinces. Sans vouloir être exhaustif, nous pouvons évoquer les cas de l'Association Orthodoxe Saint Grégoire fondée à Damas en 1912 sous les auspices du patriarcat grec-orthodoxe d'Antioche, de la communauté al-Arḍ (la Terre) créée à Homs en 1990 par le jésuite Frans van der Lugt et de l'atelier al-Manāra (le Phare), proche de l'Église grecque-melkite catholique, qui vit le jour à la fin des années 90 dans la banlieue de Damas. Ce même constat est fait à l'échelle internationale par Julia Berger, pour laquelle « les ONG chrétiennes [...] ont tendance à être liées à des structures confessionnelles et à un leadership religieux » (J. Berger, 2007 : 37). En Syrie, les raisons qui sous-tendent cette logique sont de natures diverses. Parmi elles, la marge de manœuvre relativement large dont ont bénéficié les autorités religieuses chrétiennes grâce à de bonnes relations avec le régime (L. Ruiz de Elvira, sous presse) ; également une vie sociale fortement structurée autour des Églises et, plus généralement, autour des institutions tenues par le clergé (les écoles par exemple); et, enfin, l'important capital symbolique, social et économique dont disposent fréquemment les institutions religieuses chrétiennes et leurs représentants, et ce aussi bien au Proche-Orient qu'ailleurs dans le monde (P. Bourdieu et M. de Saint Martin, 1982). À ces éléments s'ajoute un dernier facteur, à savoir l'enjeu intra-confessionnel et la compétition entre les différentes Églises qui peut être particulièrement vive dans le domaine social.

Cependant, afin de nuancer ce qui pourrait être considéré à tort comme une spécificité chrétienne, il faut préciser que, dans le cas syrien (T. Pierret, 2011 ; L. Khatib, 2011) mais aussi à l'échelle régionale (S. Ben Nefissa, 2002), cette logique ne se limite pas uniquement aux communautés chrétiennes. En effet, la fondation de nombreuses associations musulmanes sunnites à vocation sociale s'est aussi réalisée, par le passé et même dans les années 2000, en lien avec des institutions et des autorités religieuses. Citons l'exemple de Hifẓ al-Ni'ma ([Projet pour] la Préservation de la Grâce [divine]) - créé en 2002 (puis autorisé en 2006) par le charismatique et très respecté cheikh Sāriya al-Rifā'ī, prêcheur de la mosquée Zayd à Damas (T. Pierret et K. Selvik, 2009).

\section{Des associations enregistrées et des associations informelles}

Au-delà du moment fondateur, le rapport étroit établi entre les associations et les institutions chrétiennes paraît évident à la lumière du statut légal de certaines de ces structures. Nous découvrons ici des stratégies variables motivées parfois par le choix des fondateurs et des responsables associatifs, mais aussi, souvent, par les décisions des autorités religieuses elles-mêmes. En effet, si parmi 
les cinq associations évoquées plus haut, trois (Saint Vincent de Paul, Terre des Hommes Syrie et al-Mahabba) sont devenues légalement autonomes de l'Église et sont enregistrées ${ }^{11}$ auprès des autorités compétentes, à savoir le ministère des Affaires Sociales et du Travail, les deux restantes (Bayt al-Salām et al-Safīna) ont un statut particulier. Prenons le cas de Bayt al-Salām pour illustrer nos propos.

Comme cela a été précisé, Bayt al-Salām est depuis sa création sous la tutelle du patriarcat syriaque catholique qui se porte garant et veille à la bonne conduite de l'association. Les relations avec cette Église ont été bonnes par le passé. En 2009, elles étaient cependant plus tendues. Aux yeux de plusieurs de ses membres, l'archevêché ne prêtait pas assez attention à l'association et ne lui octroyait aucune aide en dehors de la mise à disposition gratuite de deux immeubles. Néanmoins, en dépit de cette relation problématique, Bayt al-Salām est resté liée de facto à ce patronage religieux à cause de son défaut de statut légal.

Malgré la flexibilité relative dont les autorités syriennes ont fait preuve durant les années 2000 (l'octroi de nouvelles autorisations devient plus souple et, à partir de l'année 2004, un processus de normalisation ${ }^{12}$ ayant pour but d'enregistrer les organisations travaillant jusque-là sans l'autorisation du ministère voit le jour), Bayt al-Salām n'a effectivement pas entrepris les procédures requises pour formaliser le passage à la légalité. A contrario, elle a choisi de rester en marge du tissu associatif enregistré. Ce statut et cette stratégie ne faisaient pourtant pas l'unanimité au sein de l'association. Si cet enregistrement n'a pas eu lieu, c'est que l'archevêché, conscient que la légalisation aurait entraîné la perte de l'emprise directe de l'Église sur l'association, n'a pas souhaité donner son accord à la direction. Pour comprendre ce type de réaction, il faut garder à l'esprit que lorsqu'une association est sous la tutelle d'une Église, elle en dépend légalement. Dans ce type de configuration, l'Église joue le rôle d'intermédiaire entre l'État et l'association et entre celle-ci et la communauté. Les dignitaires ecclésiastiques peuvent alors en tirer des bénéfices en termes de crédibilité et de visibilité et ce aussi bien auprès de la population que des autorités politiques. En revanche, lorsque l'association est enregistrée, elle n'a plus besoin de cette figure intermédiaire pour dialoguer avec les uns et les autres et le rôle de l'Église perd alors de

11. En Syrie, seules les associations autorisées par l'État sont dites « enregistrées ». En effet, avant 2011, le cadre juridique syrien diffère des cadres juridiques libanais ou palestinien, lesquels prévoient que pour qu'une association soit légalement reconnue, il suffit de la déclarer auprès des autorités compétentes (système d'enregistrement). Le système d'autorisation appliqué en Syrie s'oppose fondamentalement au système d'enregistrement selon lequel théoriquement aucune association ne peut se voir refuser son droit à exister. Dans la pratique, il équivaut à un long et ardu processus pendant lequel les membres fondateurs sont soumis à une enquête menée par les services de la Sûreté Générale.

12. Ce processus de normalisation a surtout visé les structures sunnites, perçues par le régime comme plus menaçantes que les structures chrétiennes. Ces dernières, ayant toujours bénéficié de meilleures relations avec le pouvoir en place, n'ont jamais connu les mêmes difficultés pour obtenir leur autorisation. 
l'importance. Le conseil d'administration a dès lors été contraint de rester sous la coupe d'une institution religieuse qui lui offrait théoriquement une certaine autonomie, mais avec laquelle les relations pouvaient s'avérer tendues.

En 2010, Bayt al-Salām était toujours placée sous la tutelle du patriarcat et donc considérée par les pouvoirs publics comme une activité appartenant à cette institution et non comme une organisation légale à part entière. Pour cette raison elle ne pouvait bénéficier d'aucune aide qu'elle fût de source étrangère ou étatique $^{13}$. Bayt al-Salām dépendait donc entièrement des donations privées. Ce flou dans son statut légal se reflétait dans son appellation : cette structure était définie comme étant un mašğal (atelier) - dénomination volontairement vague, moyen pour la direction de contourner le terme ğam'iyya (association), qui désigne les structures associatives autorisées par le ministère des Affaires Sociales et du Travail.

D'autres associations chrétiennes - chapeautées par d'autres Églises - se trouvaient, avant 2011, dans la même situation. Al-Safinna, al-Arḍ, les différentes branches d'Imān wa Nūr (Foi et Lumière) et l'atelier al-Manāra en constituent seulement quelques exemples. À l'évidence, leur enregistrement au ministère ne faisaient pas l'unanimité auprès des différents acteurs concernés. En réalité, nous avons répertorié deux cas de figure : d'une part, les structures qui, à l'instar de Bayt al-Salām, souhaitaient réaliser ce passage vers la légalité mais n'y parvenaient pas à cause du manque d'entente entre toutes les parties impliquées; d'autre part, celles qui refusaient le changement de statut par peur de passer sous la tutelle de l'État. Ces dernières estimaient avoir plus de marge de manœuvre (aussi bien en termes de gestion et de comptabilité qu'en termes pratiques, dans la sélection des bénéficiaires, par exemple, ou dans la planification des activités) et être plus tranquilles sous la protection de l'Église.

Pour le président de Bayt al-Salām, le grand nombre d'associations chrétiennes non enregistrées était lié au fait que les membres de ces communautés ne souhaitaient pas entrer en politique et préféraient faire profil bas. Cet argument est pourtant insuffisant étant donné que la grande majorité des associations syriennes à vocation sociale, enregistrées ou non, gardaient une distance prudente à l'égard du politique avant 2011. L'explication doit alors être cherchée ailleurs : d'une part on remarquait du côté des associations une forte volonté de s'autogérer et de rester éloignées des institutions publiques, toujours redoutées; et d'autre part on peut penser que le régime - qui s'est toujours montré conciliant et tolérant vis-à-vis des populations chrétiennes du pays - ne voyait pas dans ces structures un réel danger pour la préservation de son pouvoir. Naturellement, cette logique

13. Il faut noter ici qu'avant 2011 l'aide de l'État aux organisations enregistrées auprès du ministère des Affaires Sociales et du Travail variait en fonction de leur importance (en taille et en ressources) et de leur rapport au pouvoir. Certaines n'en recevaient aucune. 
n'est pas incompatible avec l'existence de plusieurs exceptions. Parmi elles, celle de Terre des Hommes Syrie, dont le président du conseil d'administration défendait en 2007 le choix de l'enregistrement ${ }^{14}$.

\section{De principes et pratiques aconfessionnels ...}

Comme nous venons de le montrer, un nombre important d'associations chrétiennes damascènes sont nées en lien avec des institutions religieuses, et ce dans des périodes différentes. Qui plus est, certaines d'entre elles continuaient à en dépendre directement lorsque le soulèvement populaire contre le régime de Bachar al-Assad s'est déclenché en mars 2011. Or, il est intéressant de noter qu'elles cherchent souvent à se présenter au public comme étant des structures aconfessionnelles et non-communautaires.

Lors de nos entretiens et conversations, Bayt al-Salām était définie par ses membres et son personnel comme étant une " association caritative non

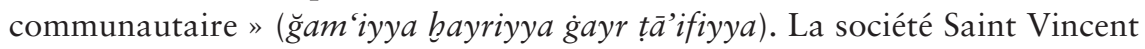
de Paul, elle aussi, était décrite par ses responsables comme étant "laïque » ('ilmāniyya) et "non communautaire " $\left(\bar{a}-t+\bar{a}^{\prime} \text { ifiyya }\right)^{15}$. Quant à Terre des Hommes Syrie, elle cherchait à s'afficher publiquement - aussi bien vis-à-vis de ses bailleurs de fonds étrangers que des autorités syriennes - comme une organisation où les considérations d'ordre religieux n'avaient pas une place prépondérante. Dans l'une de ses brochures, on pouvait lire en guise de conclusion : "Dans un pays où le "clientélisme religieux" est encore vivace et contamine les associations d'entraide, malgré le courageux effort des autorités qui travaillent pour l'enrayer, Terre des Hommes Syrie, après 25 ans de lutte, est maintenant reconnue comme Organisation Non Gouvernementale qui est ouverte à tous sans aucune étiquette ${ }^{16}$. De même, lorsque l'on visitait cette association, ses responsables tenaient à souligner le fait qu'il s'agissait bien d'une entité "ni religieuse, ni politique ", " because we don't want to be accused that we only help Christians because Père Paul is a Lazarist, so that is why we want to be very careful with that ${ }^{17}$.

Cette façon de se définir par rapport au religieux n'est nullement réservée aux contextes proche-orientaux. Bruno Duriez et ses collaborateurs font le même

14. Entretien, Damas, décembre 2007.

15. Propos extraits d'une carte de vœux de Noël envoyée par la société Saint Vincent de Paul à d'autres associations syriennes en décembre 2008.

16. Terre des Hommes Syrie, brochure informative en français et anglais.

17. Propos recueillis par Natalia Ribas-Mateos, Damas, juillet 2008. Il est intéressant de noter que, jusqu'à sa nationalisation en 1967, le collège Saint-Vincent des pères lazaristes de Damas accueillait, lui aussi, des individus issus de différentes communautés : chrétiens orthodoxes, musulmans sunnites, druzes, alaouites, juifs et même protestants (J. Bocquet, 2002). 
constat dans l'introduction de leur ouvrage consacré aux ONG confessionnelles (ONGc). "Discrétion ou nouvelle visibilité du religieux ? ", se demandent-ils. Ils y concluent : "L'édulcoration des références religieuses est bien un des constats majeurs de ces analyses sur les ONGc, en particulier occidentales » (B. Duriez et alii, 2007 : 18). Ils soulignent néanmoins que cette évolution (ou parfois cette stratégie), ne signifie pas nécessairement la perte de la référence religieuse (B. Duriez et alii, 2007 : 17).

Il est vrai qu'une partie significative des associations chrétiennes syriennes que nous avons rencontrées entre 2007 et 2010 œuvraient aussi bien pour les chrétiens que pour les musulmans. Terre des Hommes Syrie fournissait de facto un service où l'identité religieuse n'était pas un critère de discrimination dans la sélection des bénéficiaires. À ce sujet, les responsables de l'association assuraient en 2008 que " $95 \%$ des bénéficiaires sont musulmans " ${ }^{18}$. Chez Bayt al-Salām, sept des trente-trois bénéficiaires de l'année 2009 ainsi que l'un des membres du conseil d'administration étaient musulmans. Al-Safīna et al-Mahabba suivaient, elles aussi, la même ligne de travail. En outre, si l'on en croit les travaux de Géraldine Chatelard sur les tribus chrétiennes de Madaba en Jordanie, cette démarche ne serait pas exclusivement syrienne : la loi jordanienne de l'année 1953, qui n'est plus en vigueur aujourd'hui, contraignait les associations de bienfaisance chrétiennes à fournir leurs services sur une base non-confessionnelle (G. Chatelard, $2000: 216$ ).

De manière générale, cette ouverture des services aux individus issus des communautés non chrétiennes présenterait plusieurs avantages. Comme l'affirmait la directrice de Bayt al-Salām, l'absence de discrimination entre chrétiens et musulmans permettrait d'écarter toute accusation de sectarisme pouvant être proférée à l'encontre de l'association, aussi bien par les autorités syriennes que par la population, majoritairement sunnite. Ceci explique également la délicate stratégie développée par Saint Vincent de Paul au fil du temps. Celle-ci consiste à accepter un petit pourcentage de musulmans en tant que bénéficiaires non permanents (le statut de bénéficiaire permanent étant réservé exclusivement aux chrétiens) sous condition qu'ils aient adressé auparavant leur demande à une structure de bienfaisance musulmane et qu'ils aient été refusés. Ce faisant, l'association aurait réussi à trouver un équilibre et se protégerait non seulement des accusations de sectarisme mais aussi de celles de prosélytisme ${ }^{19}$.

Grâce à cette stratégie, les structures chrétiennes parviennent in fine à tirer des bénéfices d'une configuration qui, au départ, pourrait être perçue comme handicapante. En effet, en même temps qu'elles continuent de proposer des services sociaux aux communautés chrétiennes, elles contribuent à recréer des liens

18. Propos recueillis par Natalia Ribas-Mateos, Damas, juillet 2008.

19. Il faut rappeler ici qu'en 2010 plusieurs Églises évangéliques installées dans le nord du pays furent fermées par les autorités syriennes. Officieusement, elles furent accusées de prosélytisme. 
sociaux entre les populations chrétiennes et musulmanes et transmettent une image positive d'ouverture sur les autres communautés. Ceci leur permet en plus de se promouvoir aussi bien sur le plan national qu'international, notamment vis-à-vis des bailleurs de fonds.

\section{... à l'entre-soi communautaire et confessionnel}

Il n'en demeure pas moins que ces associations inscrivent leurs actions dans des réseaux de sociabilité de type communautaire tissés souvent dès la plus tendre enfance. Les écoles, les groupes de scouts, les camps d'été et les cours de catéchisme constituent autant d'espaces et d'activités où l'on fait des rencontres qui s'avèrent fréquemment durables et accompagnent l'individu tout au long de sa vie. Ces sociabilités sont par la suite renforcées, une fois l'âge adulte atteint, dans les églises - lors des messes, des baptêmes, des mariages, ...-, au sein des chorales et d'autres activités menées par et pour la communauté. C'est précisément dans cet «espace communautaire » (H. Bozarslan, 2011 : 68) que s'inscrivent et évoluent les associations ici étudiées.

Pour preuve, à Bayt al-Salām la mobilisation est fondée essentiellement sur la proximité et les relations interpersonnelles, d'autant plus qu'elle ne fait presque aucune publicité. En 2010, plusieurs employés étaient d'anciens amis de l'école, d'un groupe de scouts ou d'une chorale et se connaissaient, donc, depuis leur enfance. D'autres avaient simplement des amitiés en commun ou étaient voisins du quartier chrétien de Bāb Šarqī, situé à l'est de la vieille ville de Damas. Pour l'ensemble du personnel, l'engagement à Bayt al-Salām présentait plusieurs avantages, dont ceux de rester au sein de la communauté et de travailler à proximité de leurs foyers, situés dans les quartiers chrétiens de la capitale.

À Terre des Hommes Syrie aussi, les bénévoles, les responsables et les employés, sont majoritairement chrétiens. Ainsi, le fondateur est, nous le savons déjà, un religieux lazariste. En 2010, le président du conseil d'administration était un chrétien maronite qui a longtemps vécu en France où il enseignait la philosophie. Son cousin, lui, était le responsable du site de Kfarseta (province de Tartous). De même, celui qui dirigeait l'atelier de prothèses était un jeune chrétien qui avait fait ses études chez les pères lazaristes du Liban, dont Paul Sleiman. Pour un jeune bénévole rencontré en 2009, Terre des Hommes Syrie constituait non seulement un espace de réalisation de sa foi - dans la mesure où il y pratiquait les enseignements du Christ (la charité, c'est-à-dire l'amour de Dieu et du prochain en vue de Dieu, et le partage) - mais aussi un lieu privilégié pour " être-ensociété »(F. Adelkhah, 1998), voire en communauté - au sens où il pouvait y rencontrer, et se sociabiliser avec, ses coreligionnaires ${ }^{20}$.

20. Entretien, Tartous, juillet 2009. 
Ces mêmes logiques communautaires se trouvent à l'origine de l'engagement de certains membres de la ğam'iyyat al-Ṣalīb li-I'ānat al-Armān (Association La Croix pour l'Assistance aux Arméniens). Les propos de la directrice de Bayt al-Salām en 2010 reflètent bien ce point : Bayt al- Salām, disait-elle, n'était pas son association ; elle y travaillait, y consacrait une partie importante de son énergie et de son temps, certes, mais son association était al-Ṣalīb, une organisation de et pour la communauté arménienne, dont elle fait partie. Au moment de notre entretien, elle était membre de son assemblée générale, comme d'autres personnes de sa famille. "Être membre » - affirmait-elle - « implique de payer une cotisation mais pas seulement. Ça veut dire aussi consacrer son temps à organiser des activités, [...] Tu travailles parce que c'est ton association " ${ }^{21}$. L'engagement associatif était dans ce cas le moyen d'exprimer un fort sentiment communautaire transmis de parent à enfant.

Nos observations auprès de ces structures s’inscrivent dans un cadre général plus large de réactivation des identités confessionnelles et communautaires, y compris dans la gestion du politique (E. Picard, 2012). Selon Thierry Boissière (2005), on a assisté, dans la Syrie de Bachar al-Assad, à un retour des «liens primordiaux » (E. Picard, 2006) qui se construisent à partir des clivages ethniques et des appartenances communautaires. Ce retour s'est opéré, entre autres, au travers de l'essor de caisses d'entraide familiales ou amicales ainsi que de la multiplication d'associations caritatives fonctionnant sur la base de l'appartenance confessionnelle. Concomitamment, et découlant de ce qui précède, la Syrie a connu une consolidation du rôle joué par les hommes de religion en tant qu'intermédiaires entre ces structures, les populations bénéficiaires et les pouvoirs locaux (T. Boissière, 2005 : 150). Paolo Pinto observe également ce phénomène bien qu'il le considère plus ancien. À ce propos, il écrit que "durant les trois dernières décennies, les identités et les pratiques religieuses ont acquis, en Syrie, une importance accrue comme canaux de participation à la sphère publique, ainsi que comme cadres normatifs pour les trajectoires individuelles et les interactions sociales »(P. Pinto, 2007 : 327).

Le pouvoir baathiste, prétendument laïque, n'a donc non seulement pas empêché la persistance des clivages ethniques et religieux dans la société syrienne, mais aussi favorisé leur réactivation, notamment à partir des années 2000. Cette réactivation des "liens primordiaux » peut être lue in fine comme un échec du projet baathiste de construction nationale ${ }^{22}$; échec qui a été tragiquement mis en lumière par l'évolution violente et de plus en plus sectaire de la révolte syrienne.

21. Entretien, Damas, décembre 2009.

22. Sur l'effort de construction nationale Stéphane Valter écrit : "Ce pouvoir [baathiste] se voit ainsi contraint de jouer sur les identités, au gré des circonstances, en recourant à des représentations tantôt globalisatrices tantôt sélectives du passé, dans le dessein de construire symboliquement une communauté politique nationale lui permettant de consolider sa légitimité » (S. Valter, $2002: 13$ ). 
Enfin, outre l'inscription de ces associations dans des réseaux locaux de sociabilité communautaire, force est de constater que la religion est également très présente dans les pratiques ordinaires, l'ethos et l'imaginaire des personnes qui y sont engagées.

En effet, les références à la religion chrétienne sont omniprésentes. Elles transparaissent sous forme de citations dans les brochures et les affiches où sont intégrées soigneusement des paroles de saints et de Jésus et des citations de la Bible. Ainsi l'une des citations évoquées dans une brochure comme inspirant l'esprit et l'action de Terre des Hommes Syrie est une phrase de saint Vincent de Paul : "Fais-toi pardonner le pain que tu leur donnes » ${ }^{23}$. De la même manière, l'Association Orthodoxe Saint Grégoire affirme agir en accord avec ce qu'a dit Jésus autrefois : "J'étais affamé et vous m'avez nourri ... J'étais nu et vous m'avez habillé » ${ }^{24}$. Ces références religieuses prennent également la forme matérielle de croix et de représentations de saints affichées sur les murs des locaux. Ceci est le cas de Bayt al-Salām, où les images du Christ et de la Vierge Marie décorant les murs des deux maisons traditionnelles viennent s'ajouter aux œufs de Pâques et aux décorations de Noël des années précédentes. Ces références sont enfin véhiculées au travers de prières, de bénédictions de locaux par des prêtres, d'envois de cartes de vœux et de participations à des marchés caritatifs durant la période de Noël ou bien à travers la célébration des fêtes chrétiennes. À titre d'exemple, chez al-Safīna, les réunions de " la communauté » sont considérées comme "des moments de joie, partage et prière » ${ }^{25}$. La prière est également très présente à Bayt al-Salām où le petit déjeuner est précédé quotidiennement d'une prière collective à laquelle les bénéficiaires musulmans ne participent pas.

En somme, ces associations se nourrissent d'une forte tradition religieuse chrétienne qui modèle aussi bien les registres d'action que les valeurs et normes qui sont transmises. Au demeurant, il faut rappeler aussi que ces structures sont généralement constituées de personnes dont la foi est profonde. Cela peut être illustré par le fait que plusieurs des hommes de Bayt al-Salām, Terre des Hommes Syrie et Saint Vincent de Paul que nous avons rencontrés s'apprêtaient à devenir prêtres lorsqu'ils étaient jeunes.

\section{Insertion dans les tissus associatifs chrétiens internationaux}

Enfin, alors qu'avant 2011 une grande majorité des associations syriennes se caractérisait par une forte autonomie vis-à-vis des acteurs étrangers et que les partenariats entre organisations syriennes et organisations internationales étaient

23. Terre des Hommes Syrie, brochure informative en français et anglais.

24. Site internet de l'association : http://www.st-gos.com/ (consulté le 15 septembre 2012).

25. Site internet de l'association: http://www.larchesyria.com/fr/the-community/ (consulté le 21 février 2014). 
fortement restreints et surveillés par les autorités, une partie significative des associations chrétiennes rencontrées bénéficiait d'une bonne insertion dans des réseaux, souvent confessionnels, internationaux. Il est important de rappeler ici que le contrôle du ministère des Affaires Sociales et du Travail était très strict et que les structures associatives non enregistrées par ses soins ne pouvaient bénéficier d'aides étrangères. En outre, tout financement d'un organisme étranger à une association locale devait être validé préalablement par le ministère des Affaires Sociales et du Travail, puis par le ministère des Affaires Étrangères ${ }^{26}$.

Cette insertion privilégiée dans des réseaux internationaux se manifestait notamment sur deux plans : celui de l'accès aux ressources étrangères à l'intérieur de la Syrie, d'une part (L. Ruiz de Elvira, sous presse); celui de l'affiliation directe à des structures associatives internationales, de l'autre. A contrario, pour les associations caritatives sunnites, ce type d'affiliation à des réseaux internationaux ou occidentaux était très rare. Significativement, le réseau international Islamic Relief, qui est établi dans une centaine de pays autour du monde et plus particulièrement au Moyen-Orient (Égypte, Liban, Irak, Jordanie, Palestine et Yémen), n'était pas actif dans la Syrie des années 2000.

Ainsi, les relations entre les sociétés Saint Vincent de Paul localisées en Syrie et ailleurs dans le monde étaient toujours de mise avant 2011, comme le montrent les déclarations faites en 2009 par l'un de ses membres : « On est en contact avec Saint Vincent de Paul international. On reçoit des conseils et des directives de leur part et on les respecte tant qu'ils ne sont pas en contradiction avec la loi syrienne » ${ }^{27}$. Il en allait de même pour al-Safīna, version syrienne du réseau international "l'Arche ", qui, depuis 2011, donne régulièrement des informations sur la situation des «amis " de l'Arche de Damas : " A lot of people have been asking for news of the members of L'Arche in Damascus, wanting to show their support: we are very grateful to you! Our friends in Al Safīna regularly tell us just how much these thoughts and prayers mean to them, and how much they feel linked with the rest of the Federation ${ }^{28}$. Jean Vanier, fondateur du réseau, leur adressait un message en décembre 2013 : « Mes chers Amis, Voici la lettre que j'ai adressée à tous les amis chrétiens du Moyen-Orient à la demande du journal catholique La Croix. Nous, en France, nous vivons Noël dans la paix, mais il y a tous ceux qui vivent Noël dans la souffrance et la guerre. Je vous

26. Outre la volonté du régime d'entraver la coopération entre acteurs locaux et internationaux et de contrôler de près tous les projets, l'absence de financements étrangers s'explique aussi par deux autres éléments : d'une part la méfiance et la peur des responsables des associations à l'égard des bailleurs de fonds étrangers, d'autre part le manque d'intérêt, la méfiance et, parfois, le mépris des acteurs étrangers vis-à-vis des projets caritatifs syriens.

27. Entretien, Damas, octobre 2009.

28. Site de l'Arche Canada: http://www.larchecommons.ca/en/national/news/news_of_ larche_in_syria_2012-07-30 (consulté le 15 avril 2014). 
transmets cette lettre pour que nous puissions prier ensemble les uns pour les autres " ${ }^{29}$. Terre des Hommes Syrie, enfin, est non seulement membre du réseau international Terre des Hommes (la FITdH, fondée en $1966^{30}$ ), mais coopère aussi régulièrement avec plusieurs organisations et organismes internationaux d'origine confessionnelle, dont Caritas, l'International Catholic Migration Commission, le Secours Catholique, l'American Friends Service Committee (une organisation de la communauté quaker des États-Unis) et la Délégation Catholique pour la Coopération, laquelle a envoyé à plusieurs reprises des spécialistes (en psychomotricité et en orthophonie) bénévoles à Terre des Hommes Syrie.

L'insertion dans ces réseaux contribue non seulement à rendre visibles les structures associatives chrétiennes au-delà des frontières syriennes mais facilite aussi l'arrivée de bénévoles venant d'Europe ou d'ailleurs et favorise les partenariats avec les ONG étrangères installées en Syrie. Ainsi, certaines associations chrétiennes bénéficient de moyens d'action et de reconnaissance bien plus importants que beaucoup d'associations musulmanes. Sur un plan spirituel, ces échanges servent également à rapprocher les communautés chrétiennes d'Orient de celles d'Occident, renforçant de cette manière le sentiment d'appartenance à un christianisme mondial. Ces derniers aspects s'avèrent être d'une importance fondamentale dans un environnement qui reste majoritairement musulman.

\section{***:}

Tout en ayant fréquemment recours à une rhétorique insistant sur leur caractère aconfessionnel et non-communautaire et tout en faisant montre d'une réelle ouverture des services aux populations musulmanes, les associations chrétiennes que nous avons connues en Syrie dans les années 2000 entretiennent un rapport étroit et profond au religieux, révélateur d'un repli communautaire. L'empreinte religieuse varie d'une structure à l'autre, prenant parfois la forme d'une dépendance directe aux institutions chrétiennes, d'autres fois celle d'une relation plus souple et moins visible au premier abord. L'ensemble de ces associations s'appuient pourtant sur des réseaux confessionnels tissés dans la durée à plusieurs niveaux - le local, le régional et l'international - et contribuent sans doute à maintenir, voire à renforcer, le lien communautaire. Le référent religieux ne peut ainsi que transparaître au sein de ces structures, et ce aussi bien dans les pratiques que dans les discours, les normes et les valeurs.

Enfin, les identités confessionnelles et communautaires étudiées dans cet article doivent être replacées dans un mouvement général de réactivation des liens confessionnels, ethniques et localistes opéré sous le régime de Bachar al-Assad

29. Idem.

30. La première association Terre des Hommes est fondée en 1960 en Suisse. 
et signant l'échec de la construction d'une société nationale. L'enracinement religieux et, pour ce qui est des populations chrétiennes, l'attachement aux différentes Églises, s'inscrivent donc dans une logique plus large de communautarisation de la société syrienne, et cela bien avant 2011.

Laura RUIZ DE ELVIRA

Centre for Near and Middle East Studies (CNMS) Marburg University, Germany laura.ruizdeelvira@uni-marburg.de

\section{Bibliographie}

Adelkhah Fariba, 1998, Etre moderne en Iran, Paris, Karthala.

Association de Bienfaisance Saint Vincent de Paul de Damas, 2003, Dalīl al-Manșūrū, (Guide de la Société Saint Vincent de Paul), Damas.

BERGER Julia, 2007, « Les organisations non gouvernementales religieuses. Quelques pistes de recherche", in Duriez B. et alii (dir.), Les ONG confessionnelles. Religions et action internationale, Paris, L'Harmattan, Collection Religion en questions, p. 23-40.

Ben Nefissa Sarah (dir.), 2002, Pouvoirs et associations dans le monde arabe, Paris, Éditions CNRS.

Bocquet Jérôme, 2002, Le collège Saint-Vincent des pères lazaristes de Damas : l'enseignement français en Syrie (1864-1967), thèse de doctorat en histoire, Université Paris I Sorbonne.

BoIssière Thierry, 2005, "Précarité économique, instabilité de l'emploi et pratiques sociales en Syrie ", REMMM, 105-106, p. 109-131.

Bozzo Anna et Luizard Pierre-Jean (dir.), 2011, Les sociétés civiles dans le monde musulman, Paris, La Découverte.

Boukhaima Soukaina, 2002, "Le mouvement associatif en Syrie ", in Ben Nefissa S. (dir.), Pouvoirs et associations dans le monde arabe, Paris, Éditions CNRS, p. 77-94.

Bourdieu Pierre et DE SAINT Martin Monique, 1982, "La sainte famille », Actes de la recherche en sciences sociales, vol. 44-45, p. 2-53.

Bourdieu Pierre, 1984/2002, Questions de sociologie, Paris, Les Éditions de Minuit.

Bozarslan Hamit, 2011, Sociologie politique du Moyen Orient, Paris, La Découverte.

Chatelard Géraldine, 2000, Briser la mosaïque : lien social et identités collectives chez les chrétiens de Madaba, Jordanie 1870-1997, thèse de doctorat à l'EHESS, Paris.

-, 2004, Briser la mosaïque. Les tribus chrétiennes de Madaba, Jordanie, XIXe-XX ${ }^{e}$ siècles, Paris, CNRS Éditions.

Cholvy Gérard, 2003, Frédéric Ozanam. L'engagement d'un intellectuel catholique au $\mathrm{XIX}$ siècle, Paris, Fayard.

DuRIEZ Bruno et alii, 2007, "Introduction ", in Duriez B. et alii (dir.), Les ONG confessionnelles. Religions et action internationale, Paris, L'Harmattan, Collection Religion en questions, p. 9-19.

Griffith Sidney H., 2008, The Church in the Shadow of the Mosque: Christians and Muslims in the World of Islam, Princeton et Oxford, Princeton University Press.

Hashmi Sohail H. (ed.), 2002, Islamic Political Ethics. Civil society, Pluralism, and Conflict, Princeton, Princeton University Press. 
Кнатів Line, 2011, Islamic Revivalism in Syria: the Rise and Fall of Ba'thist Secularism, Londres et New York, Routledge.

McCallum Fiona, 2010, Christian Religious Leadership in the Middle East: The Political Role of the Patriarch, Lewiston, Edwin Mellen Press.

Norton Richard Augustus (éd.), 1995, Civil Society in the Middle East, Leiden et New York, E. J. Brill.

PICARD Elizabeth, 2006, "Les liens primordiaux, vecteurs de dynamiques politiques ", in Picard E. (dir.), La Politique dans le monde arabe, Paris, Armand Colin, p. 55-77.

-, 2012, "Nation-Building and Minority Rights in the Middle East ", in Longva A. N. et Roald A.S. (éd.), Religious Minorities in the Middle East. Domination, SelfEmpowerment, Accommodation, Leiden et Boston, Brill.

PICHON Frédéric, 2010, Maaloula (XIXe-XXI siècles), Du vieux avec du neuf, Histoire et identité d'un village chrétien de Syrie, Beyrouth, Presses de l'IFPO.

PierRet Thomas et Selvik Kjetil, 2009, "Limits of "Authoritarian Upgrading” in Syria: Private Welfare, Islamic Charities, and the Rise of the Zayd movement ", IJMES, vol. 41, no 4, p. 595-614.

-, 2011, Baas et Islam en Syrie, Paris, PUF.

PINTo Paolo, 2007, "Religions et religiosité en Syrie ", in Dupret B. et alii (dir.), La Syrie au présent. Reflets d'une société, Paris, Actes Sud, p. 325-358.

Ruiz de Elvira Laura, 2010, "L'État syrien de Bachar al-Assad à l'épreuve des ONG », Maghreb Machrek, no 203, p. 41-57.

-, 2012a, "Agir dans les marges. Portrait d'une association caritative chrétienne en Syrie ", in Abu-Sada C. et Challand B. (dir.), Le développement, une affaire d'ONG ?, Paris, Karthala-IFPO-IREMAM, p. 41-67.

-, 2012b, "Les catégories à l'épreuve des “associations réelles" : ni tout à fait "laïques" et développementalistes, ni tout à fait confessionnelles et de bienfaisance. Étude de cas de l'association Terre des Hommes Syrie ", A Contrario, 18, p. 79-96.

,- 2015 , "Christian charities and the Ba'thist Regime in Bashar al-Asad's Syria. A comparative analysis ", in Stenberg L. et Salamandra C. (éd.), Syria from reform to revolt (Volume 2): Religion, Society, and Culture under Bashar al-Asad, Syracuse NY, Syracuse University Press.

- et ZINTL Tina, 2012, Civil Society and the State in Syria: The Outsourcing of Social Responsibility, Boulder, Lynne Rienner.

LE SAUX Mathieu, 2006, "Les dynamiques contradictoires du champ associatif syrien ", REMMM, no 115-116, p. 193-209.

TAHA Zakaria, 2012, Le parti Baath et la dynastie al-Asad en Syrie: la laïcité dans un contexte communautaire, texte de la communication présentée lors d'une conférence tenue à Rennes le 6 juin, disponible sur http://halshs.archives-ouvertes.fr/docs/00/73/ 47/73/PDF/Le_parti_Baath_et_la_dynastie_al-Asad_en_Syrie_la_laA_citA_dans_un_ contexte_communautaire.pdf

Terre des Hommes Syrie, A brief extract for 41 years service for the handicaped, document non publié et non daté.

Valognes Jean-Pierre, 1994, Vie et mort des chrétiens d'Orient, Paris, Fayard.

VALTER Stéphane, 2002, La construction nationale syrienne: légitimation de la nature communautaire du pouvoir par le discours historique, Paris, Centre national de la recherche scientifique Éditions. 


\section{Les associations chrétiennes de Damas et leurs rapports variables au religieux (2000-2010)}

Malgré le recours fréquent à une rhétorique insistant sur leur caractère aconfessionnel et non-communautaire et malgré une réelle ouverture des services aux populations musulmanes, les associations chrétiennes à vocation sociale de Damas entretiennent un rapport étroit à la religion chrétienne. Celui-ci varie d'une structure à l'autre, prenant parfois la forme d'une dépendance directe par rapport aux institutions chrétiennes, d'autres fois celle d'une relation plus souple et moins visible au premier abord. L'ensemble de ces associations s'appuient pourtant sur des réseaux confessionnels tissés dans la durée à plusieurs niveaux - le local, le régional et l'international et contribuent sans doute à renforcer le lien communautaire. Le référent religieux ne peut ainsi que transparaître au sein de ces structures, et ce aussi bien dans les pratiques que dans les discours, les normes et les valeurs.

Mots-clés: Syrie, associations à vocation sociale, chrétiens arabes, rapport au religieux, confessionnalisme, communautarisme.

\section{Christian Associations in Damascus and their Fluctuant Relations to Religion (2000-2010)}

In spite of the frequent use of a rhetoric insisting on their non-confessional and noncommunity-oriented character, and in spite of a real opening of their services towards the Muslim populations, socially oriented Christian associations arguably entertain a close relationship with the Christian Religion. This relationship fluctuates from a structure to another, taking sometimes the shape of a direct dependence towards Christian institutions, sometimes the shape of a more flexible and at first sight less visible relationship. Yet all of these associations lean on confessional networks weaved on a long-term scale on several levels - local, regional, international - and by way of this, certainly contribute to reinforcing communal ties. Thus the religious reference appears in the midst of these structures, throughout the practices as well as throughout the discourses, norms and values.

Key words: Syria, social organization, Arab Christians, relationship to religion, sectarianism.

\section{Las asociaciones cristianas de Damasco y sus relaciones variables con lo religioso (2000-2010)}

A pesar del recurso frecuente a una retórica insistente sobre su carácter aconfesional y no-comunitario, y más allá de una real apertura de los servicios a la población musulmana, las asociaciones cristianas con vocación social de Damasco sostienen una relación estrecha con la religión cristiana. Esto varía de una estructura a la otra, tomando a veces la forma de una dependencia directa a las instituciones cristianas, $y$ otras la de una relación más laxa y menos visible en principio. El conjunto de estas asociaciones se apoya sin embargo en redes confesionales tejidas en el largo plazo en varios niveles - local, regional e internacional - y contribuyen sin duda a reforzar el lazo comunitario. El referente religioso aparece entonces en el interior de estas estructuras, tanto en las prácticas como en los discursos, las normas y los valores.

Palabras clave: Siria, asociación social, cristianos árabes, relación con lo religioso, confesionalismo, comunitario. 